\title{
Fixed points in uniform spaces
}

\author{
Phichet Chaoha* and Sittichoke Songsa-ard
}

\author{
${ }^{*}$ Correspondence: \\ phichet.c@chula.ac.th \\ Department of Mathematics and \\ Computer Science, Faculty of \\ Science, Chulalongkorn University, \\ Bangkok, 10330, Thailand
}

\begin{abstract}
We improve Angelov's fixed point theorems of $\Phi$-contractions and $j$-nonexpansive maps in uniform spaces and investigate their fixed point sets using the concept of virtual stability. Some interesting examples and an application to the solution of a certain integral equation in locally convex spaces are also given.
\end{abstract}

Keywords: fixed points; $\Phi$-contractions; uniform spaces

\section{Introduction}

In 1987 [1], Angelov introduced the notion of $\Phi$-contractions on Hausdorff uniform spaces, which simultaneously generalizes the well-known Banach contractions on metric spaces as well as $\gamma$-contractions [2] on locally convex spaces, and he proved the existence of their fixed points under various conditions. Later in 1991 [3], he also extended the notion of $\Phi$-contractions to $j$-nonexpansive maps and gave some conditions to guarantee the existence of their fixed points. However, there is a minor flaw in his proof of Theorem 1 [3] where the surjectivity of the map $j$ is implicitly used without any prior assumption. Additionally, we observe that such a map $j$ can be naturally replaced by a multi-valued map $J$ to obtain a more general, yet interesting, notion of $J$-nonexpansiveness. Therefore, in this work, we aim to correct and simplify the proof of Theorem 1 [3] as well as extend the notion of $j$-nonexpansive maps to $J$-nonexpansive maps and investigate the existence of their fixed points. Then we introduce $J$-contractions, a special kind of $J$-nonexpansive maps, that play the similar role as Banach contractions in yielding the uniqueness of fixed points. With the notion of $J$-contractions, we are able to recover results on $\Phi$-contractions proved in [1] as well as present some new fixed point theorems in which one of them naturally leads to a new existence theorem for the solution of a certain integral equation in locally convex spaces. Finally, we prove that, under a mild condition, $J$-nonexpansive maps are always virtually stable in the sense of [4] and hence their fixed point sets are retracts of their convergence sets. An example of a virtually stable $J$-nonexpansive map whose fixed point set is not convex is also given.

\section{Fixed point theorems}

For any set $S$, we will use $\mathcal{P}^{f}(S)$ and $|S|$ to denote the set of all nonempty finite subsets of $S$ and the cardinality of $S$, respectively. Let $(E, \mathcal{A})$ be a Hausdorff uniform space whose uniformity is generated by a saturated family of pseudometrics $\mathcal{A}=\left\{d_{\alpha}: \alpha \in A\right\}$ indexed by $A$, $\emptyset \neq X \subseteq E$, and $J: A \rightarrow \mathcal{P}^{f}(A)$. Interested readers should consult [5] for general topological concepts of uniform spaces, and [6] for the complete development of fixed point theory in

@2014 Chaoha and Songsa-ard; licensee Springer. This is an Open Access article distributed under the terms of the Creative Commons Attribution License (http://creativecommons.org/licenses/by/2.0), which permits unrestricted use, distribution, and reproduction in any medium, provided the original work is properly cited. 
uniform spaces that motivates this work. We first give the definition of a $J$-nonexpansive map as follows:

Definition 2.1 A self-map $T: X \rightarrow X$ is said to be $J$-nonexpansive if for each $\alpha \in A$,

$$
d_{\alpha}(T x, T y) \leq \sum_{\beta \in J(\alpha)} d_{\beta}(x, y)
$$

for any $x, y \in X$.

Example 2.2 Let $1<p<\infty, E=\ell_{p}$ be equipped with the weak topology, and $T: \ell_{p} \rightarrow \ell_{p}$ be defined by

$$
T\left(x_{1}, x_{2}, \ldots\right)=\left(\frac{\left|x_{1}+x_{3}\right|}{3}, \frac{\left|x_{2}+x_{4}\right|}{3}, x_{3}, x_{4}, \ldots\right),
$$

for any $\left(x_{1}, x_{2}, \ldots\right) \in \ell_{p}$. Then $\mathcal{A}=\left\{|f|: f \in \ell_{p}^{*}\right\}$, where $|f|(x)=|f(x)|$ for each $x \in \ell_{p}$.

By Theorem 4.6 in [7], we have

$$
\begin{aligned}
|f(T x-T y)| \leq & \left|\frac{\|f\|}{3}\left(x_{1}-y_{1}+x_{3}-y_{3}\right)\right|+\left|\frac{\|f\|}{3}\left(x_{2}-y_{2}+x_{4}-y_{4}\right)\right| \\
& +|| f\left\|\left(x_{1}-y_{1}\right)|+|\right\| f \|\left(x_{2}-y_{2}\right)|+| f(x-y) \mid,
\end{aligned}
$$

for each $f \in \ell_{p}^{*}, x=\left(x_{1}, x_{2}, \ldots\right) \in \ell_{p}$ and $y=\left(y_{1}, y_{2}, \ldots\right) \in \ell_{p}$. Here, $\|f\|=\sup \{|f(x)|: x \in$ $X,\|x\| \leq 1\}$.

By letting $J: \ell_{p}^{*} \rightarrow \mathcal{P}^{f}\left(\ell_{p}^{*}\right)$ be defined by $J(f)=\left\{|f|,\left|g_{1}\right|,\left|g_{2}\right|,\left|g_{3}\right|,\left|g_{4}\right|\right\}$, for each $f \in \ell_{p}^{*}$, where

$$
g_{1}(x)=\frac{\|f\|}{3}\left(x_{1}+x_{3}\right), \quad g_{2}(x)=\frac{\|f\|}{3}\left(x_{2}+x_{4}\right), \quad g_{3}(x)=\|f\| x_{1}, \quad g_{4}(x)=\|f\| x_{2},
$$

for each $x=\left(x_{1}, x_{2}, \ldots\right) \in \ell_{p}$, it follows that $T$ is $J$-nonexpansive.

The above definition of a $J$-nonexpansive map clearly extends the definition of a $j$-nonexpansive map in [3]. Before giving general existence criteria for fixed points of $J$-nonexpansive maps, we need the following notations. For each $\alpha \in A$ and $n \in \mathbf{N}$, we let

$$
A_{n}(\alpha)=\left\{\left(\alpha_{1}, \ldots, \alpha_{n}\right): \alpha_{1} \in J(\alpha) \text { and } \alpha_{k} \in J\left(\alpha_{k-1}\right) \text { for } 1<k \leq n\right\}
$$

and

$$
A(\alpha)=\left\{\left(\alpha_{1}, \alpha_{2}, \ldots\right): \alpha_{1} \in J(\alpha) \text { and } \alpha_{k} \in J\left(\alpha_{k-1}\right) \text { for } k>1\right\} .
$$

When there is no ambiguity, we will denote an element of both $A_{n}(\alpha)$ and $A(\alpha)$ simply by $\left(\alpha_{k}\right)$. Notice that for each $\alpha \in A$ and $n \in \mathbf{N}$, the sets $A_{n}(\alpha)$ and $\pi_{n}(A(\alpha))$ are finite, where $\pi_{n}$ denotes the $n$th coordinate projection $\left(\alpha_{k}\right) \mapsto \alpha_{n}$.

Lemma 2.3 Every J-nonexpansive map is continuous. 
Proof Suppose $T: X \rightarrow X$ is $J$-nonexpansive. Let $x \in X$ and $\left(x_{\gamma}\right)$ be a net in $X$ converging to $x$. Then for each $\alpha \in A$, we have

$$
d_{\alpha}\left(T x_{\gamma}, T x\right) \leq \sum_{\beta \in J(\alpha)} d_{\beta}\left(x_{\gamma}, x\right)
$$

Since $\left(x_{\gamma}\right)$ converges to $x,\left(d_{\beta}\left(x_{\gamma}, x\right)\right)$ converges to 0 for any $\beta \in A$, and this proves the continuity of $T$.

Theorem 2.4 Let $T: X \rightarrow X$ be J-nonexpansive whose $A(\alpha)$ is finite for any $\alpha \in A$. Then $T$ has a fixed point in $X$ if and only if there exists $x_{0} \in X$ such that

(i) the sequence $\left(T^{n} x_{0}\right)$ has a convergence subsequence, and

(ii) for each $\alpha \in A$ and $\left(\alpha_{k}\right) \in A(\alpha), \lim _{n \rightarrow \infty} d_{\alpha_{n}}\left(x_{0}, T x_{0}\right)=0$.

Proof $(\Rightarrow)$ : It is obvious by letting $x_{0}$ be a fixed point of $T$.

$(\Leftarrow)$ : Suppose that $\left(T^{n_{i}} x_{0}\right)$ converges to some $z \in X$. Let $\alpha \in A$ and $\left(\alpha_{k}\right) \in A(\alpha)$. Then $\lim _{i \rightarrow \infty} d_{\alpha}\left(z, T^{n_{i}} x_{0}\right)=0$ and $\lim _{n \rightarrow \infty} d_{\alpha_{n}}\left(x_{0}, T x_{0}\right)=0$. We can choose $N \in \mathbf{N}$ sufficiently large so that $d_{\alpha}\left(z, T^{n_{i}} x_{0}\right)<\epsilon$ and $d_{\alpha_{n_{i}}}\left(x_{0}, T x_{0}\right)<\epsilon$, for all $i \geq N$. It follows that

$$
\begin{aligned}
d_{\alpha}\left(z, T^{n_{i}+1} x_{0}\right) & \leq d_{\alpha}\left(z, T^{n_{i}} x_{0}\right)+d_{\alpha}\left(T^{n_{i}} x_{0}, T^{n_{i}}\left(T x_{0}\right)\right) \\
& \leq d_{\alpha}\left(z, T^{n_{i}} x_{0}\right)+\sum_{\left(\alpha_{k}\right) \in A_{n_{i}}(\alpha)} d_{\alpha_{n_{i}}}\left(x_{0}, T x_{0}\right) \\
& \leq(1+|A(\alpha)|) \epsilon .
\end{aligned}
$$

Since $\alpha$ is arbitrary, $\left(T^{n_{i}+1} x_{0}\right)$ converges to $z$. By the continuity of $T$, we have $z=T z$ and hence $z$ is a fixed point of $T$.

As a corollary of the previous theorem, we immediately obtain Theorem 1 [3], with a corrected and simplified proof, as follows:

Corollary 2.5 Let $T: X \rightarrow X$ be a j-nonexpansive map. If there exists $x_{0} \in X$ such that

(i) the sequence $\left(T^{n} x_{0}\right)$ has a convergence subsequence, and

(ii) for every $\alpha \in A, \lim _{n \rightarrow \infty} d_{j^{n}(\alpha)}\left(x_{0}, T x_{0}\right)=0$,

then $T$ has a fixed point.

Proof The proof follows directly from the previous theorem by considering the map $J$ : $\alpha \mapsto\{j(\alpha)\}$. Notice that $A(\alpha)=\left\{\left(j^{n}(\alpha)\right)\right\}$ which is finite.

We will now consider a special kind of $J$-nonexpansive maps that resemble Banach contractions in yielding the uniqueness of fixed points. Let $\Phi$ denote the family of all functions $\phi:[0, \infty) \rightarrow[0, \infty)$ satisfying the following conditions:

$(\Phi 1) \phi$ is non-decreasing and continuous from the right, and

(Ф2) $\phi(t)<t$ for any $t>0$.

Notice that $\phi(0)=0$, and we will call $\phi \in \Phi$ subadditive if $\phi\left(t_{1}+t_{2}\right) \leq \phi\left(t_{1}\right)+\phi\left(t_{2}\right)$ for all $t_{1}, t_{2} \geq 0$. Also, for a subfamily $\left\{\phi_{\alpha}\right\}_{\alpha \in A}$ of $\Phi, \alpha \in A,\left(\alpha_{k}\right) \in A_{n}(\alpha)$ and $i \leq n$, we let

$$
\phi_{\left(\alpha_{k}\right)}^{i}=\phi_{\alpha_{1}} \circ \cdots \circ \phi_{\alpha_{i}}
$$


Definition 2.6 A self-map $T: X \rightarrow X$ is said to be a $J$-contraction if for each $\alpha \in A$, there exists $\phi_{\alpha} \in \Phi$ such that

$$
d_{\alpha}(T x, T y) \leq \sum_{\beta \in J(\alpha)} \phi_{\alpha}\left(d_{\beta}(x, y)\right)
$$

for any $x, y \in X$, and $\phi_{\alpha}$ is subadditive whenever $|J(\alpha)|>1$.

Clearly, a $\Phi$-contraction as defined in [1] is a $J$-contraction and a $J$-contraction is always $J$-nonexpansive. A natural example of a $J$-contraction can be obtained by adding (finitely many) appropriate $\Phi$-contractions as shown in the following example.

Example 2.7 Given two $\Phi$-contractions $T_{1}: X \rightarrow X$ and $T_{2}: X \rightarrow X$ as defined [1]. Then there exist $j_{1}, j_{2}: A \rightarrow A$, and for each $\alpha \in A$, there exist $\phi_{1, \alpha}, \phi_{2, \alpha} \in \Phi$ such that

$$
d_{\alpha}\left(T_{1} x, T_{1} y\right) \leq \phi_{1, \alpha}\left(d_{j_{1}(\alpha)}(x, y)\right) \quad \text { and } \quad d_{\alpha}\left(T_{2} x, T_{2} y\right) \leq \phi_{2, \alpha}\left(d_{j_{2}(\alpha)}(x, y)\right),
$$

for any $\alpha \in A$ and $x, y \in X$. If for each $\alpha \in A, j_{1}(\alpha) \neq j_{2}(\alpha)$ and there is a subadditive $\phi_{3, \alpha} \in \Phi$ so that $\phi_{1, \alpha}(t) \leq \phi_{3, \alpha}(t)$ and $\phi_{2, \alpha}(t) \leq \phi_{3, \alpha}(t)$ for any $t \geq 0$, then the map $H=T_{1}+T_{2}$ is clearly a $J$-contraction with respect to $J(\alpha)=\left\{j_{1}(\alpha), j_{2}(\alpha)\right\}$ and $\phi_{H, \alpha}=\phi_{3, \alpha}$ for any $\alpha \in A$.

Lemma 2.8 If $T: X \rightarrow X$ is a J-contraction. Then we have

$$
d_{\alpha}\left(T^{n} x, T^{n} y\right) \leq \sum_{\left(\alpha_{k}\right) \in A_{n}(\alpha)} \phi_{\alpha} \circ \phi_{\left(\alpha_{k}\right)}^{n-1}\left(d_{\alpha_{n}}(x, y)\right)
$$

for any $\alpha \in A, n \geq 2$ and $x, y \in X$.

Proof Recall that $\phi_{\alpha}$ is assumed to be subadditive whenever $|J(\alpha)|>1$. Then, for any $\alpha \in A$, $n \geq 2$ and $x, y \in X$, we clearly have

$$
\begin{aligned}
d_{\alpha}\left(T^{n} x, T^{n} y\right) \leq & \sum_{\alpha_{1} \in J(\alpha)} \phi_{\alpha}\left(d_{\alpha_{1}}\left(T^{n-1} x, T^{n-1} y\right)\right) \\
\leq & \sum_{\alpha_{1} \in J(\alpha)} \phi_{\alpha}\left(\sum_{\alpha_{2} \in J\left(\alpha_{1}\right)} \phi_{\alpha_{1}}\left(d_{\alpha_{2}}\left(T^{n-2} x, T^{n-2} y\right)\right)\right) \\
\leq & \sum_{\alpha_{1} \in J(\alpha)} \sum_{\alpha_{2} \in J\left(\alpha_{1}\right)} \phi_{\alpha} \circ \phi_{\alpha_{1}}\left(d_{\alpha_{2}}\left(T^{n-2} x, T^{n-2} y\right)\right) \\
& \vdots \\
\leq & \sum_{\alpha_{1} \in J(\alpha)} \sum_{\alpha_{2} \in J\left(\alpha_{1}\right)} \cdots \sum_{\alpha_{n} \in J\left(\alpha_{n-1}\right)} \phi_{\alpha} \circ \phi_{\alpha_{1}} \circ \cdots \circ \phi_{\alpha_{n-1}}\left(d_{\alpha_{n}}(x, y)\right) \\
& =\sum_{\left(\alpha_{k}\right) \in A_{n}(\alpha)} \phi_{\alpha} \circ \phi_{\left(\alpha_{k}\right)}^{n-1}\left(d_{\alpha_{n}}(x, y)\right) .
\end{aligned}
$$

We now obtain some general criteria for the existence of fixed points of $J$-contractions.

Theorem 2.9 Suppose $X$ is sequentially complete and $T: X \rightarrow X$ is a J-contraction whose $A(\alpha)$ is finite for any $\alpha \in A$. If $T$ satisfies the following conditions: 
(i) for each $\alpha \in A$, there exists $c_{\alpha} \in \Phi$ such that

$$
\phi_{\alpha_{i}}(t) \leq c_{\alpha}(t)
$$

$$
\text { for any }\left(\alpha_{k}\right) \in A(\alpha), i \in \mathbf{N}, t \geq 0 \text {, and }
$$

(ii) there exists $x_{0} \in X$ such that for each $\alpha \in A,\left(\alpha_{k}\right) \in A(\alpha), i \in \mathbf{N}$ and $n, m \in \mathbf{N}$, we have

$$
d_{\alpha_{i}}\left(T^{n} x_{0}, T^{m} x_{0}\right) \leq M_{\alpha}\left(x_{0}\right)
$$

for some $M_{\alpha}\left(x_{0}\right) \in \mathbf{R}$,

then $T$ has a fixed point. Moreover, iffor each $\alpha \in A$ and $x, y \in X$, there exists $F_{\alpha}(x, y) \in \mathbf{R}_{0}^{+}$ such that

$$
d_{\alpha_{i}}(x, y) \leq F_{\alpha}(x, y)
$$

for all $\left(\alpha_{k}\right) \in A(\alpha)$ and $i \in \mathbf{N}$, then the fixed point of $T$ is unique.

Proof For each $\alpha \in A$ and $n, m, N \in \mathbf{N}$, since $\phi_{\alpha}$ is non-decreasing, we have

$$
\begin{aligned}
d_{\alpha}\left(T^{n} x_{0}, T^{m} x_{0}\right) & \leq \sum_{\alpha_{1} \in J(\alpha)} \phi_{\alpha}\left(d_{\alpha_{1}}\left(T^{n-1} x_{0}, T^{m-1} x_{0}\right)\right) \\
& \leq \sum_{\alpha_{1} \in J(\alpha)} \phi_{\alpha}\left(\sup \left\{d_{\alpha_{1}}\left(T^{n-1} x_{0}, T^{m-1} x_{0}\right): n, m \geq N\right\}\right),
\end{aligned}
$$

and by letting $h_{N}^{\alpha}:=\sup \left\{d_{\alpha}\left(T^{n} x_{0}, T^{m} x_{0}\right): n, m \geq N\right\}$, it follows that

$$
\begin{aligned}
h_{N}^{\alpha} & \leq \sum_{\alpha_{1} \in J(\alpha)} \phi_{\alpha}\left(\sup \left\{d_{\alpha_{1}}\left(T^{n-1} x_{0}, T^{m-1} x_{0}\right): n, m \geq N\right\}\right) \\
& =\sum_{\alpha_{1} \in J(\alpha)} \phi_{\alpha}\left(h_{N-1}^{\alpha_{1}}\right) \\
& \leq \sum_{\alpha_{1} \in J(\alpha)} \sum_{\alpha_{2} \in J\left(\alpha_{1}\right)} \phi_{\alpha}\left(\phi_{\alpha_{1}}\left(h_{N-2}^{\alpha_{2}}\right)\right) \\
& \vdots \\
\leq & \sum_{\left(\alpha_{k}\right) \in A_{N-1}(\alpha)} \phi_{\alpha} \circ \phi_{\left(\alpha_{k}\right)}^{N-1}\left(h_{1}^{\alpha_{N-1}}\right) \\
\leq & \sum_{\left(\alpha_{k}\right) \in A_{N-1}(\alpha)} c_{\alpha}^{N}\left(M_{\alpha}\left(x_{0}\right)\right) \\
\leq & |A(\alpha)| c_{\alpha}^{N}\left(M_{\alpha}\left(x_{0}\right)\right) .
\end{aligned}
$$

Also, for a given $t \geq 0$, since $0 \leq c_{\alpha}^{N}(t)=c_{\alpha}\left(c_{\alpha}^{N-1}(t)\right)<c_{\alpha}^{N-1}(t)$, we have $\lim _{N \rightarrow \infty} c_{\alpha}^{N}(t)=r_{\alpha}$ for some $r_{\alpha} \geq 0$. Since $c_{\alpha}$ is right continuous, we have $\lim _{N \rightarrow \infty} c_{\alpha}\left(c_{\alpha}^{N-1}(t)\right)=c_{\alpha}\left(r_{\alpha}\right)$, and hence $c_{\alpha}\left(r_{\alpha}\right)=r_{\alpha}$. Therefore, $r_{\alpha}=0$. By (1), it follows that $\lim _{N \rightarrow \infty} h_{N}^{\alpha}=0$. Since $\alpha$ is arbitrary, $\left(T^{k} x_{0}\right)$ is a Cauchy sequence and, by sequential completeness, converges to some $z \in X$. Notice also that $z$ must be a fixed point of $T$ by continuity. 
Now suppose that for each $x, y \in X$ and $\alpha \in A$, there exists $F_{\alpha}(x, y) \in \mathbf{R}_{0}^{+}$such that $d_{\alpha_{i}}(x, y) \leq F_{\alpha}(x, y)$ for all $\left(\alpha_{k}\right) \in A(\alpha)$ and $i \in \mathbf{N}$. If $x, y$ are fixed points of $T$, then by Lemma 2.8, we have for each $\alpha \in A$ and $n \in \mathbf{N}$,

$$
\begin{aligned}
d_{\alpha}(x, y) & =d_{\alpha}\left(T^{n} x, T^{n} y\right) \\
& \leq \sum_{\left(\alpha_{k}\right) \in A_{n}(\alpha)} \phi_{\alpha} \circ \phi_{\left(\alpha_{k}\right)}^{n-1}\left(d_{\alpha_{n}}(x, y)\right) \\
& \leq \sum_{\left(\alpha_{k}\right) \in A_{n}(\alpha)} c_{\alpha}^{n}\left(d_{\alpha_{n}}(x, y)\right) \\
& \leq|A(\alpha)| c_{\alpha}^{n}\left(F_{\alpha}(x, y)\right) .
\end{aligned}
$$

Since $\lim _{n \rightarrow \infty} c_{\alpha}^{n}\left(F_{\alpha}(x, y)\right)=0$, we must have $x=y$.

As a corollary of the previous theorem, we immediately obtain Theorem 1 in [1] as follows.

Corollary 2.10 Suppose $X$ is a bounded and sequentially complete subset of $E$ and $T: X \rightarrow$ $X$ is $\Phi$-contraction. If

(i) for each $\alpha \in A$, there exists $c_{\alpha} \in \Phi$ such that $\phi_{j^{n}(\alpha)}(t) \leq c_{\alpha}(t)$ for all $n \in \mathbf{N}$ and $t \geq 0$,

(ii) for each $n \in \mathbf{N}$, $\sup \left\{d_{j^{n}(\alpha)}(x, y): x, y \in X\right\} \leq p(\alpha):=\sup \left\{d_{\alpha}(x, y): x, y \in X\right\}$,

then there exists a unique fixed point $x \in X$ of $T$.

Proof For each $x_{0}, x, y \in X, \alpha \in A,\left(\alpha_{k}\right) \in A(\alpha)$ and $i, m, n \in \mathbf{N}$, by letting $J(\alpha)=\{j(\alpha)\}$ and $M_{\alpha}\left(x_{0}\right)=p(\alpha)=F_{\alpha}(x, y)$, we have $A(\alpha)=\left\{\left(\alpha, j(\alpha), j^{2}(\alpha), \ldots, j^{k}(\alpha), \ldots\right)\right\}, d_{\alpha_{i}}\left(T^{m} x_{0}, T^{n} x_{0}\right)=$ $d_{j^{i}(\alpha)}\left(T^{m} x_{0}, T^{n} x_{0}\right) \leq M_{\alpha}\left(x_{0}\right)$ and $d_{\alpha_{i}}(x, y) \leq F_{\alpha}(x, y)$. Hence, by Theorem 2.9, $T$ has a unique fixed point.

Theorem 2.11 Suppose $X$ is sequentially complete and $T: X \rightarrow X$ is a self-map satisfying: for each $\alpha \in A$ and $k \in \mathbf{N}$, there exist $\phi_{\alpha, k} \in \Phi$, a finite set $D_{\alpha, k}$ and a map $P_{\alpha, k}: D_{\alpha, k} \rightarrow A$ such that

$$
d_{\alpha}\left(T^{k} x, T^{k} y\right) \leq \sum_{\gamma \in D_{\alpha, k}} \phi_{\alpha, k}\left(d_{P_{\alpha, k}(\gamma)}(x, y)\right)
$$

for any $x, y \in X$.

1. If there exists $x_{0} \in X$ such that for each $\alpha \in A$ there exists $M_{\alpha}\left(x_{0}\right) \in \mathbf{R}_{0}^{+}$so that $\sum_{k \in \mathbf{N}}\left|D_{\alpha, k}\right| \phi_{\alpha, k}\left(M_{\alpha}\left(x_{0}\right)\right)<\infty$ and

$$
d_{P_{\alpha, k}(\gamma)}\left(x_{0}, T x_{0}\right) \leq M_{\alpha}\left(x_{0}\right)
$$

for all $k \in \mathbf{N}$ and $\gamma \in D_{\alpha, k}$, then $T$ has a fixed point in $X$.

2. If for each $\alpha \in A$ and $x, y \in X$, there exists $F_{\alpha}(x, y) \in \mathbf{R}_{0}^{+}$such that

$$
\sum_{k \in \mathbf{N}}\left|D_{\alpha, k}\right| \phi_{\alpha, k}\left(F_{\alpha}(x, y)\right)<\infty \text { and }
$$

$$
d_{P_{\alpha, k}(\gamma)}(x, y) \leq F_{\alpha}(x, y),
$$


for all $k \in \mathbf{N}$ and $\gamma \in D_{\alpha, k}$, then $T$ has a unique fixed point in $X$ and, for any $x \in X$, the sequence $\left(T^{n} x\right)$ converges to the fixed point of $T$.

Proof First notice that $T$ is clearly a $J$-contraction.

1. For any $\alpha \in A$ and $m>n \in \mathbf{N}$, we have

$$
\begin{aligned}
d_{\alpha}\left(T^{n} x_{0}, T^{m} x_{0}\right) & \leq \sum_{n \leq i<m} d_{\alpha}\left(T^{i} x_{0}, T^{i+1} x_{0}\right) \\
& \leq \sum_{n \leq i<m} \sum_{\gamma \in D_{\alpha}, i} \phi_{\alpha, i}\left(d_{P_{\alpha, i}(\gamma)}\left(x_{0}, T x_{0}\right)\right) \\
& \leq \sum_{n \leq i<m}\left|D_{\alpha, i}\right| \phi_{\alpha, i}\left(M_{\alpha}\left(x_{0}\right)\right) .
\end{aligned}
$$

Also, since $\sum_{k \in \mathbf{N}}\left|D_{\alpha, k}\right| \phi_{\alpha, k}\left(M_{\alpha}\left(x_{0}\right)\right)<\infty,\left(T^{k} x_{0}\right)$ is a Cauchy sequence and converges to a fixed point of $T$ by the sequential completeness of $X$ and the continuity of $T$.

2. For any $x \in X, \alpha \in A$ and $m>n \in \mathbf{N}$, we have

$$
\begin{aligned}
d_{\alpha}\left(T^{n} x, T^{m} x\right) & \leq \sum_{n \leq i<m} d_{\alpha}\left(T^{i} x, T^{i+1} x\right) \\
& \leq \sum_{n \leq i<m} \sum_{\gamma \in D_{\alpha}, i} \phi_{\alpha, i}\left(d_{P_{\alpha, i}(\gamma)}(x, T x)\right) \\
& \leq \sum_{n \leq i<m}\left|D_{\alpha, i}\right| \phi_{\alpha, i}\left(F_{\alpha}(x, T x)\right) .
\end{aligned}
$$

Also, since $\sum_{k \in \mathbf{N}}\left|D_{\alpha, k}\right| \phi_{\alpha, k}\left(F_{\alpha}(x, T x)\right)<\infty,\left(T^{k} x\right)$ is a Cauchy sequence and converges to a fixed point of $T$ by the sequential completeness of $X$ and the continuity of $T$.

Now, since for each $\alpha \in A, k \in \mathbf{N}$ and $x, y \in F(T)$,

$$
\begin{aligned}
d_{\alpha}(x, y) & =d_{\alpha}\left(T^{k} x, T^{k} y\right) \\
& \leq \sum_{\gamma \in D_{\alpha, k}} \phi_{\alpha, k}\left(d_{P_{\alpha, k}(\gamma)}(x, y)\right) \\
& \leq \sum_{\gamma \in D_{\alpha, k}} \phi_{\alpha, k}\left(F_{\alpha}(x, y)\right) \\
& =\left|D_{\alpha, k}\right| \phi_{\alpha, k}\left(F_{\alpha}(x, y)\right),
\end{aligned}
$$

and $\lim _{k \rightarrow \infty}\left|D_{\alpha, k}\right| \phi_{\alpha, k}\left(F_{\alpha}(x, y)\right)=0$, we have the uniqueness.

Corollary 2.12 (Theorem 5 in [1]) Let us suppose

(i) for each $\alpha \in A$ and $n>0$, there exist $\phi_{\alpha, n} \in \Phi$ and $j(\alpha, n) \in A$ such that

$$
d_{\alpha}\left(T^{n} x, T^{n} y\right) \leq \phi_{\alpha, n}\left(d_{j(\alpha, n)}(x, y)\right)
$$

for any $x, y \in X$,

(ii) there exists $x_{0} \in X$ such that $d_{j(\alpha, n)}\left(x_{0}, T x_{0}\right) \leq p(\alpha)<\infty(n=1,2, \ldots)$, $\sum_{n} \phi_{\alpha, n}(p(\alpha))<\infty$ and $j: A \times \mathbf{N} \rightarrow A$.

Then $T$ has at least one fixed point in $X$. 
Proof By letting $D_{\alpha, k}=\{j(\alpha, k)\}$ for any $\alpha \in A$ and $k \in \mathbf{N}$ and $P_{\alpha, k}=\left.\pi_{k}\right|_{D_{\alpha, k}}$. Then for each $i \in \mathbf{N}$, we have $\left|D_{\alpha, i}\right|=1$ and $M_{\alpha}\left(x_{0}\right)=p(\alpha)$. By Theorem 2.11(2), $T$ has a fixed point.

Theorem 2.13 Suppose $X$ is sequentially complete and $T: X \rightarrow X$ is a J-contraction whose $A(\alpha)$ is finite for each $\alpha \in A$. If, for each $\alpha \in A$, there exists $c_{\alpha} \in \Phi$ satisfying:

(i) $c_{\alpha}(t) / t$ is non-decreasing in $t$,

(ii) $\phi_{\alpha_{n}}(t) \leq c_{\alpha}(t)$ for any $\left(\alpha_{k}\right) \in A(\alpha), n \in \mathbf{N}$ and $t \in[0, \infty)$, and

(iii) there exist $x_{0} \in X$ and $M_{\alpha}\left(x_{0}\right) \in \mathbf{R}^{+}$such that $d_{\alpha_{n}}\left(x_{0}, T x_{0}\right) \leq M_{\alpha}\left(x_{0}\right)$ for any $\left(\alpha_{k}\right) \in A(\alpha)$ and $n \in \mathbf{N}$,

then $T$ has a fixed point in $X$.

Proof Let $D_{\alpha, i}=A_{i}(\alpha), P_{\alpha, i}\left(\left(\alpha_{k}\right)\right)=\alpha_{i}$, and $\phi_{\alpha, i}(t)=c_{\alpha}^{i}(t)$ for any $i \in \mathbf{N}, \alpha \in A,\left(\alpha_{k}\right) \in A_{i}(\alpha)$, and $t \in[0, \infty)$. Then for any $\alpha \in A$ and $x, y \in X$, we have, by Lemma 2.8 ,

$$
\begin{aligned}
d_{\alpha}\left(T^{i} x, T^{i} y\right) & \leq \sum_{\left(\alpha_{k}\right) \in A_{i}(\alpha)} \phi_{\alpha} \circ \phi_{\left(\alpha_{k}\right)}^{i-1}\left(d_{\alpha_{i}}(x, y)\right) \\
& \leq \sum_{\left(\alpha_{k}\right) \in A_{i}(\alpha)} c_{\alpha}^{i}\left(d_{\alpha_{i}}(x, y)\right) \\
& =\sum_{\left(\alpha_{k}\right) \in D_{\alpha, i}} \phi_{\alpha, i}\left(d_{P_{\alpha, i}\left(\left(\alpha_{k}\right)\right)}(x, y)\right) .
\end{aligned}
$$

Since

$$
\frac{\left|D_{\alpha, i+1}\right| \phi_{\alpha, i+1}\left(M_{\alpha}\left(x_{0}\right)\right)}{\left|D_{\alpha, i}\right| \phi_{\alpha, i}\left(M_{\alpha}\left(x_{0}\right)\right)}=\frac{\left|A_{i+1}(\alpha)\right| c_{\alpha}^{i+1}\left(M_{\alpha}\left(x_{0}\right)\right)}{\left|A_{i}(\alpha)\right| c_{\alpha}^{i}\left(M_{\alpha}\left(x_{0}\right)\right)} \leq \frac{c_{\alpha}\left(c_{\alpha}^{i}\left(M_{\alpha}\left(x_{0}\right)\right)\right)}{c_{\alpha}^{i}\left(M_{\alpha}\left(x_{0}\right)\right)} \leq \frac{c_{\alpha}\left(M_{\alpha}\left(x_{0}\right)\right)}{M_{\alpha}\left(x_{0}\right)}<1,
$$

for any $i \in \mathbf{N}$, we have $\sum_{i \in \mathbf{N}}\left|D_{\alpha, i}\right| \phi_{\alpha, i}\left(M_{\alpha}\left(x_{0}\right)\right)<\infty$. Then by Theorem 2.11(1), $T$ has a fixed point.

Corollary 2.14 (Theorem 2 in [1]) Let us suppose

(i) the operator $T: X \rightarrow X$ is a $\Phi$-contraction,

(ii) for each $\alpha \in A$ there exists a $\Phi$-function $c_{\alpha}$ such that $\phi_{j^{n}(\alpha)}(t) \leq c_{\alpha}(t)$ for all $n \in \mathbf{N}$ and $c_{\alpha}(t) / t$ is non-decreasing,

(iii) there exists an element $x_{0} \in X$ such that $d_{j^{n}(\alpha)}\left(x_{0}, T x_{0}\right) \leq p(\alpha)<\infty(n=1,2, \ldots)$.

Then $T$ has at least one fixed point in $X$.

Proof By letting $J(\alpha)=\{j(\alpha)\}$ for any $\alpha \in A$ and $M_{\alpha}\left(x_{0}\right)=p(\alpha)$. Then $|A(\alpha)|=1$, and, by Theorem 2.13, $T$ has a fixed point.

Example 2.15 Given a sequentially complete locally convex space $X$, and two $\Phi$ contractions $T_{1}, T_{2}: X \rightarrow X$; i.e., there exist $j_{1}, j_{2}: A \rightarrow A$, and for each $\alpha \in A$, there exist $\phi_{1, \alpha}, \phi_{2, \alpha} \in \Phi$ such that

$$
d_{\alpha}\left(T_{1} x, T_{1} y\right) \leq \phi_{1, \alpha}\left(d_{j_{1}(\alpha)}(x, y)\right) \quad \text { and } \quad d_{\alpha}\left(T_{2} x, T_{2} y\right) \leq \phi_{2, \alpha}\left(d_{j_{2}(\alpha)}(x, y)\right)
$$

for any $\alpha \in A$ and $x, y \in X$. Suppose further that 
(i) $j_{1}^{n+1}=j_{2}^{n} \circ j_{1}$ and $j_{1}^{n} \circ j_{2}=j_{2}^{n+1}$ for any $n \in \mathbf{N}$,

(ii) for each $\alpha \in A, \phi_{1, \alpha}(t)=c_{1}(\alpha) t$ and $\phi_{2, \alpha}(t)=c_{2}(\alpha) t$ for some $c_{1}(\alpha)+c_{2}(\alpha) \in(0,1)$, and

(iii) there exists $x_{0} \in X$ such that $d_{j_{1}^{n}(\alpha)}\left(x_{0}, T_{1} x_{0}\right) \leq p_{1}\left(x_{0}, \alpha\right)<\infty$ and $d_{j_{2}^{n}(\alpha)}\left(x_{0}, T_{2} x_{0}\right) \leq p_{2}\left(x_{0}, \alpha\right)<\infty$ for any $\alpha \in A$ and $n=1,2, \ldots$.

Then $H=\frac{T_{1}+T_{2}}{2}$ is a $J$-contraction with $J(\alpha)=\left\{j_{1}(\alpha), j_{2}(\alpha)\right\}$ and $\phi_{H, \alpha}(t)=\left(c_{1}(\alpha)+c_{2}(\alpha)\right) t$. Also, by (i) and (iii), we have $|A(\alpha)|=2<\infty$ and

$$
d_{\alpha_{n}}\left(x_{0}, H x_{0}\right) \leq \frac{d_{\alpha_{n}}\left(x_{0}, T_{1} x_{0}\right)+d_{\alpha_{n}}\left(x_{0}, T_{2} x_{0}\right)}{2} \leq \frac{p_{1}\left(x_{0}, \alpha\right)+p_{2}\left(x_{0}, \alpha\right)}{2} .
$$

Hence, $H$ satisfies all conditions in Theorem 2.13, and it has a fixed point in $X$. Notice that $H$ may not be a $\Phi$-contraction, by choosing $j_{1}$ and $j_{2}$ so that $d_{j_{1}(\alpha)}+d_{j_{2}(\alpha)} \notin \mathcal{A}$ for some $\alpha \in A$, and hence Theorem 2 in [1] cannot be applied.

We now end this section by giving an application to the solution of a certain integral equation in locally convex spaces.

Example 2.16 Following terminologies in [8], let $X$ be an $\mathcal{S}$-space topologized by the family of seminorms $\left\{|\cdot|_{\alpha}: \alpha \in A\right\}$ and $C([0, T] ; X)$ the space of all continuous functions from $[0, T]$ into $X$ topologized by the family of seminorms $\left\{\|\cdot\|_{\alpha}: \alpha \in A\right\}$, where $\|x\|_{\alpha}:=$ $\sup _{t \in[0, T]}|x(t)|_{\alpha}$ for any $x \in C([0, T] ; X)$. Let $L(X)$ denote the set of all continuous linear operators on $X$,

$$
L_{0}(X)=\left\{l \in L(X): \forall \alpha \in A, \exists M(\alpha)>0, \forall x \in X,|l x|_{\alpha} \leq M(\alpha)|x|_{\alpha}\right\},
$$

and let $\{S(t)\}_{t \geq 0}$ be a $C_{0}$-semigroup on $X$ such that $S:[0, \infty) \rightarrow L_{0}(X)$ is locally bounded.

Now, we replace $\mathrm{H} 3$ and $\mathrm{H} 5$ in [8] by conditions (N1), (N2) and (N3) as follows:

(N1) $B: C([0, T] ; X) \rightarrow C([0, T] ; X)$ is an operator such that there exists $J_{B}: A \rightarrow \mathcal{P}^{f}(A)$ so that for any $\alpha \in A$, there is $k_{\alpha, B} \in L_{\mathrm{loc}}^{1}([0, T] ;[0, \infty))$ such that

$$
|B x(t)-B y(t)|_{\alpha} \leq k_{\alpha, B}(t) \sum_{\beta \in J_{B}(\alpha)}|x(t)-y(t)|_{\beta},
$$

for any $x, y \in C([0, T] ; X)$.

(N2) $f:[0, T] \times X \times X \rightarrow X$ is continuous and there exist $J_{f}: A \rightarrow \mathcal{P}^{f}(A)$ and $K_{f} \in L_{\mathrm{loc}}^{1}([0, T] ;[0, \infty))$ such that for each $\alpha \in A$,

$$
\left|f\left(t, u_{1}, v_{1}\right)-f\left(t, u_{2}, v_{2}\right)\right|_{\alpha} \leq K_{f}(t)\left(\sum_{\beta \in J_{f}(\alpha)}\left|u_{1}-u_{2}\right|_{\beta}+\left|v_{1}-v_{2}\right|_{\alpha}\right),
$$

for any $t \in[0, T]$ and $u_{1}, u_{2}, v_{1}, v_{2} \in X$,

(N3) $K_{f} \cdot k_{\alpha, B} \in L_{\text {loc }}^{1}([0, T] ;[0, \infty))$.

Consider the integral equation

$$
x(t)=S(t) x_{0}+\int_{0}^{t} S(t-s) f(s, x(s), B x(s)) d s ; \quad t \in[0, T]
$$


whose solution is closely related to the mild solution of the differential equation

$$
\frac{d x}{d t}=a x+f(t, x(t), B x(t))
$$

where $a$ denotes the infinitesimal generator of $\{S(t)\}_{t \geq 0}$.

We now define an operator $G$ on $C_{x_{0}}([0, T] ; X)=\left\{x \in C([0, T] ; X): x(0)=x_{0}\right\}$ by

$$
(G x)(t)=S(t) x_{0}+\int_{0}^{t} S(t-s) f(s, x(s), B x(s)) d s,
$$

for any $x \in C_{x_{0}}([0, T] ; X)$. Following the proof of Theorem 3 in [8] and for each $t>0$, $S(t) \in L_{0}(X)$, then we can show that, for any $\alpha \in A$, there exists $M(\alpha)>0$ such that

$$
\|G x-G y\|_{\alpha} \leq H_{\alpha}\left(\sum_{\beta \in J_{f}(\alpha)}\|x-y\|_{\beta}+\sum_{\beta \in J_{B}(\alpha)}\|x-y\|_{\beta}\right),
$$

where $H_{\alpha}=\max \left\{M(\alpha) \int_{0}^{T} K_{f}(s) d s, M(\alpha) \int_{0}^{T} K_{f}(s) k_{\alpha, B}(s) d s\right\}$. It is easy to see that if for each $\alpha \in A, H_{\alpha} \in(0,1)$ and $J_{f}(\alpha) \cap J_{B}(\alpha)=\emptyset$, then $G$ is a $J$-contraction with $J_{G}(\alpha)=J_{f}(\alpha) \cup J_{B}(\alpha)$.

In particular, if we assume further that for each $\alpha \in A, J_{f}(\alpha)=\{\alpha\},\left|J_{B}(\alpha)\right|=1$ such that $J_{B} \circ J_{B}=J_{B}$ and $H_{\alpha}=H_{J_{B}(\alpha)}<\frac{1}{2}$. Then for any $k \in \mathbf{N}$ and $x, y \in C_{x_{0}}([0, T] ; X)$, we have

$$
\begin{aligned}
\left\|G^{k} x-G^{k} y\right\|_{\alpha} & \leq H_{\alpha}^{k}\|x-y\|_{\alpha}+\left(\sum_{i=1}^{k}\left(2 H_{J_{B}(\alpha)}\right)^{k-i} H_{\alpha}^{i}\right)\|x-y\|_{J_{B}(\alpha)} \\
& =H_{\alpha}^{k}\|x-y\|_{\alpha}+\left(\sum_{i=1}^{k} 2^{k-i} H_{\alpha}^{k}\right)\|x-y\|_{J_{B}(\alpha)} \\
& \leq 2^{k-1} H_{\alpha}^{k}\left(\|x-y\|_{\alpha}+\sum_{i=1}^{k}\|x-y\|_{J_{B}(\alpha)}\right) .
\end{aligned}
$$

Now, by letting $\phi_{\alpha, k}(t)=2^{k-1} H_{\alpha}^{k} t, D_{\alpha, k}=\left\{(1, \alpha),\left(1, J_{B}(\alpha)\right)\left(2, J_{B}(\alpha)\right), \ldots,\left(k, J_{B}(\alpha)\right)\right\}, P_{\alpha, k}(\gamma)=$ $\pi_{2}(\gamma)$, and $F_{\alpha}(x, y)=\max \left\{\|x-y\|_{\alpha},\|x-y\|_{B_{B}(\alpha)}\right\}$, we have

(i) $\|x-y\|_{P_{\alpha, k}(\gamma)} \leq F_{\alpha}(x, y)$ for any $x, y \in C_{x_{0}}([0, T] ; X), k \in \mathbf{N}, \alpha \in A$, and $\gamma \in D_{\alpha, k}$,

(ii) $\sum_{k \in \mathbf{N}}\left|D_{\alpha, k}\right| \phi_{\alpha, k}\left(F_{\alpha}(x, y)\right)=\sum_{k \in \mathbf{N}} \frac{k+1}{2}\left(2 H_{\alpha}\right)^{k} F_{\alpha}(x, y)<\infty$ for any $x, y \in C_{x_{0}}([0, T] ; X)$ and $\alpha \in A$.

Therefore, by Theorem 2.11(2), $G$ has a unique fixed point, so the integral equation (2) has a unique solution.

\section{Fixed point sets}

In this section, we will show that, under a mild condition, a $J$-nonexpansive map is always virtually stable. This immediately gives a connection between the fixed point set and the convergence set of a $J$-nonexpansive map. Recall that a continuous self-map $T: X \rightarrow X$, whose fixed point set $F(T)$ is nonempty, on a Hausdorff space $X$ is said to be virtually stable [4] if for each $x \in F(T)$ and each neighborhood $U$ of $x$, there exist a neighborhood $V$ of $x$ and an increasing sequence $\left(k_{n}\right)$ of positive integers such that $T^{k_{n}}(V) \subseteq U$ for all $n \in \mathbf{N}$. When the sequence $\left(k_{n}\right)$ is independent of the point $x$ and the neighborhood $U$, we simply call $T$ a uniformly virtually stable map with respect to $\left(k_{n}\right)$. For example, a (quasi-) 
nonexpansive self-map, whose fixed point set is nonempty, on a metric space is always uniformly virtually stable with respect to the sequence $(n)$ of all natural numbers. An important feature of a virtually stable map is the connection between its fixed point set and its convergence set as given in the following theorem.

Theorem 3.1 ([4], Theorem 2.6) Suppose $X$ is a regular space. If $T: X \rightarrow X$ is virtually stable, then $F(T)$ is a retract of $C(T)$, where $C(T)$ is the (Picard) convergence set of $T$ defined as follows:

$$
C(T)=\left\{x \in X: \text { the sequence }\left(T^{n} x\right) \text { converges }\right\} .
$$

As in the previous section, let $(E, \mathcal{A})$ be a Hausdorff uniform space whose uniformity is generated by a saturated family of pseudometrics $\mathcal{A}=\left\{d_{\alpha}: \alpha \in A\right\}$ indexed by $A$ and $\emptyset \neq$ $X \subseteq E$. The following theorem gives a general criterion for a self-map on $X$ to be virtually stable.

Theorem 3.2 Let $T: X \rightarrow X$ be a self-map whose fixed point set $F(T)$ is nonempty, and which satisfies the following conditions:

(i) for each $\alpha \in A$ and $k \in \mathbf{N}$, there exist a finite set $D_{\alpha, k}$ and a map $P_{\alpha, k}: D_{\alpha, k} \rightarrow A$ such that

$$
d_{\alpha}\left(T^{k} x, T^{k} y\right) \leq \sum_{\gamma \in D_{\alpha, k}} d_{P_{\alpha, k}(\gamma)}(x, y)
$$

for any $x, y \in X$,

(ii) there exists $N \in \mathbf{N}$ such that $\left|D_{\alpha, n}\right| \leq\left|D_{\alpha, N}\right|$ and $P_{\alpha, n}\left(D_{\alpha, n}\right) \subseteq P_{\alpha, N}\left(D_{\alpha, N}\right)$ for any $n \geq N$ and $\alpha \in A$.

Then $T$ is uniformly virtually stable with respect to the sequence of all natural numbers.

Proof Let $z \in F(T)$ and let $U$ be a neighborhood of $z$. We may assume that $U=\bigcap_{i=1}^{m}\{w \in$ $\left.X: d_{\alpha_{i}}(w, z)<\epsilon\right\}$ for some $\epsilon>0$ and $\alpha_{1}, \ldots, \alpha_{m} \in A$. For each $n \in \mathbf{N}$, let

$$
V_{n}=\bigcap_{i=1}^{m} \bigcap_{\gamma \in D_{\alpha_{i}, n}}\left\{w \in X: d_{P_{\alpha_{i}, n}(\gamma)}(w, z)<\frac{\epsilon}{\left|D_{\alpha_{i}, n}\right|}\right\} .
$$

By (ii), there exists $N \in \mathbf{N}$ such that $\left|D_{\alpha_{i}, n}\right| \leq\left|D_{\alpha_{i}, N}\right|$ and $P_{\alpha_{i}, n}\left(D_{\alpha_{i}, n}\right) \subseteq P_{\alpha_{i}, N}\left(D_{\alpha_{i}, N}\right)$ for any $n \geq N$ and $i=1, \ldots, m$. Let $V=V_{1} \cap V_{2} \cap \cdots \cap V_{N}$ which is clearly a nonempty open subset of $X, y \in V, l \in \mathbf{N}$ and $i \in\{1, \ldots, m\}$. It follows that

$$
d_{\alpha_{i}}\left(T^{l} y, z\right)=d_{\alpha_{i}}\left(T^{l} y, T^{l} z\right) \leq \sum_{\gamma \in D_{\alpha_{i}, l}} d_{P_{\alpha_{i}}, l}(\gamma)(y, z)
$$

If $l<N$, then

$$
d_{\alpha_{i}}\left(T^{l} y, z\right)<\sum_{\gamma \in D_{\alpha_{i}, l}} \frac{\epsilon}{\left|D_{\alpha_{i}, l}\right|}=\epsilon
$$




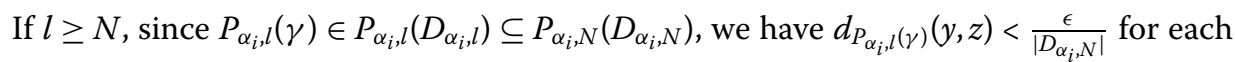
$\gamma \in D_{\alpha_{i}, l}$, and hence

$$
d_{\alpha_{i}}\left(T^{l} y, z\right)<\sum_{\gamma \in D_{\alpha_{i}, l}} \frac{\epsilon}{\left|D_{\alpha_{i}, N}\right|}=\frac{\epsilon\left|D_{\alpha_{i},}\right|}{\left|D_{\alpha_{i}, N}\right|} \leq \epsilon .
$$

Hence, $T$ is uniformly virtually stable with respect to the sequence of all natural numbers.

Corollary 3.3 Suppose that $T$ is J-nonexpansive with $F(T) \neq \emptyset$. If there exists $N \in \mathbf{N}$ such that $\left|A_{n}(\alpha)\right| \leq\left|A_{N}(\alpha)\right|$ and $\pi_{n}\left(A_{n}(\alpha)\right) \subseteq \pi_{N}\left(A_{N}(\alpha)\right)$ for any $n \geq N$ and $\alpha \in A$, then $T$ is uniformly virtually stable with respect to the sequence of all natural numbers.

Proof By letting $D_{\alpha, n}=A_{n}(\alpha)$ and $P_{\alpha, n}=\left.\pi_{n}\right|_{A_{n}(\alpha)}$ for any $n \in \mathbf{N}$ and $\alpha \in A$, we have

$$
d_{\alpha}\left(T^{l} x, T^{l} y\right) \leq \sum_{\gamma \in D_{\alpha, l}} d_{P_{\alpha, l}(\gamma)}(x, y),
$$

for any $x, y \in X$. The result then follows from Theorem 3.2.

Example 3.4 Let $E=\ell_{2}$ equipped with the weak topology and $T: \ell_{2} \rightarrow \ell_{2}$ be defined by

$$
T\left(x_{1}, x_{2}, \ldots\right)=\left(\frac{\left|x_{1}+x_{3}\right|}{3}, \frac{\left|x_{2}+x_{4}\right|}{3}, x_{3}, x_{4}, \ldots\right),
$$

for any $\left(x_{1}, x_{2}, \ldots\right) \in \ell_{2}$. Then $\mathcal{A}=\left\{|f|: f \in \ell_{2}\right\}$, and by Lemma 4.5 and Theorem 4.6 in [7], we have

$$
\begin{aligned}
& \left|f\left(T^{n} x-T^{n} y\right)\right| \\
& \leq 2\|f\|\left[\frac{\sqrt{2}}{9}\left(\left|x_{1}-y_{1}+x_{3}-y_{3}\right|+\left|x_{2}-y_{2}+x_{4}-y_{4}\right|\right)\right. \\
& \left.\quad+\frac{\sqrt{2}\left(\left|x_{1}-y_{1}\right|+\left|x_{2}-y_{2}\right|+\left|x_{1}-y_{1}+x_{3}-y_{3}\right|+\left|x_{2}-y_{2}+x_{4}-y_{4}\right|\right)}{9-6 \sqrt{2}}\right] \\
& \quad+\|f\|\left(\frac{1}{3}\left|x_{1}-y_{1}\right|+\left|x_{1}-y_{1}+x_{3}-y_{3}\right|+\frac{1}{3}\left|x_{2}-y_{2}\right|+\left|x_{2}-y_{2}+x_{4}-y_{4}\right|\right) \\
& \quad+\|f\|\left|x_{1}-y_{1}\right|+\|f\|\left|x_{2}-y_{2}\right|+|f(x-y)|,
\end{aligned}
$$

for each $f \in \ell_{2}, n \in \mathbf{N}, x=\left(x_{1}, x_{2}, \ldots\right)$ and $y=\left(y_{1}, y_{2}, \ldots\right) \in \ell_{2}$.

By letting $J: \ell_{2} \rightarrow \mathcal{P}\left(\ell_{2}\right)$ be defined by $J(f)=\left\{|f|,\left|g_{1}\right|,\left|g_{2}\right|,\left|g_{3}\right|,\left|g_{4}\right|\right\}$ for each $f \in \ell_{2}$, where

$$
\begin{aligned}
& g_{1}(x)=\|f\|\left(\frac{2 \sqrt{2}}{9}+\frac{2 \sqrt{2}}{9-6 \sqrt{2}}+1\right)\left(x_{1}+x_{3}\right), \\
& g_{2}(x)=\|f\|\left(\frac{2 \sqrt{2}}{9}+\frac{2 \sqrt{2}}{9-6 \sqrt{2}}+1\right)\left(x_{2}+x_{4}\right), \\
& g_{3}(x)=\|f\|\left(\frac{2 \sqrt{2}}{9-6 \sqrt{2}}+\frac{4}{3}\right) x_{1}, \quad g_{4}(x)=\|f\|\left(\frac{2 \sqrt{2}}{9-6 \sqrt{2}}+\frac{4}{3}\right) x_{2},
\end{aligned}
$$

for each $x=\left(x_{1}, x_{2}, \ldots\right) \in \ell_{2}$, it follows that $T$ is $J$-nonexpansive. 
Notice that $(0,0, \ldots)$ is a fixed point of $T$, and for each $f \in \ell_{2}$ and $n, m \in \mathbf{N}, \pi_{n}(A(|f|))=$ $\pi_{m}(A(|f|))$. Then, by Theorem 3.2, $T$ is virtually stable and hence the fixed point set of $T$ is a retract of the convergence set of $T$. Moreover, the fixed point set is not convex because $x=(1,1,2,2,0, \ldots)$ and $y=(1,1,-4,-4,0, \ldots)$ are fixed points of $T$, while the convex combination $\frac{1}{2} x+\frac{1}{2} y=(1,1,-1,-1,0, \ldots)$ is not.

Competing interests

The authors declare that they have no competing interests.

Authors' contributions

All authors contributed equally to the writing of this paper. All authors read and approved the final manuscript.

\section{Acknowledgements}

The authors are grateful to the anonymous referee(s) for their valuable comments and suggestions for improving this manuscript. This research is (partially) supported by the Centre of Excellence in Mathematics, the Commission on Higher Education, Thailand.

Received: 23 January 2014 Accepted: 23 May 2014 Published: 02 Jun 2014

\section{References}

1. Angelov, VG: Fixed point theorems in uniform spaces and applications. Czechoslov. Math. J. 37, 19-33 (1987)

2. Cain, GL, Nashed, MZ: Fixed points and stability for a sum of two operators in locally convex spaces. Pac. J. Math. 39(3), 581-592 (1971)

3. Angelov, VG: J-Nonexpansive mappings in uniform spaces and applications. Bull. Aust. Math. Soc. 43, 331-339 (1991)

4. Chaoha, P, Atiponrat, W: Virtually stable maps and their fixed point sets. J. Math. Anal. Appl. 359, 536-542 (2009)

5. Weil, A: Sur les Espaces à Structure Uniforme et la Topologie Générale. Hermann, Paris (1938)

6. Angelov, VG: Fixed Points in Uniform Spaces and Applications. Cluj University Press, Cluj-Napoca (2009)

7. Chaoha, P, Songsa-ard, S: Fixed points of functionally Lipschitzian maps. J. Nonlinear Convex Anal. 15(4), 665-679 (2014)

8. Chonwerayuth, A, Termwuttipong, I, Chaoha, P: Piecewise continuous mild solutions of a system governed by impulsive differential equations in locally convex spaces. Science Asia 37, 360-369 (2011)

10.1186/1687-1812-2014-134

Cite this article as: Chaoha and Songsa-ard: Fixed points in uniform spaces. Fixed Point Theory and Applications 2014, 2014:134

\section{Submit your manuscript to a SpringerOpen ${ }^{\circ}$ journal and benefit from:}

$\rightarrow$ Convenient online submission

- Rigorous peer review

Immediate publication on acceptance

Open access: articles freely available online

- High visibility within the field

- Retaining the copyright to your article 